\title{
Deporte y social media: el caso de la Primera División del fútbol español
}

\author{
Joaquín SoTELO GoNZÁLEZ \\ Universidad Complutense de Madrid \\ joaquin.sotelo@ccinf.ucm.es
}

Recibido: 10 de junio de 2012.

Aceptado: 11 de octubre de 2012.

\begin{abstract}
Resumen
Examinamos el papel que las nuevas tecnologías de la información y la comunicación y los usos asociados a ellas están desempeñando en el ámbito del deporte, más en concreto, en el de la Liga de Fútbol Profesional española. Este estudio muestra algunos de los notables cambios que las antedichas tecnologías han provocado en las estructuras comunicativas del deporte. Durante los últimos años y dentro de un proceso que continúa, el mundo del deporte también ha integrado en su vertiente comunicativa las nuevas tecnologías y los medios sociales promovidos por éstas.
\end{abstract}

Palabras clave: Deporte; comunicación; medios sociales; fútbol; España; tecnología.

\section{Sport and Social Media: Spain’s “Primera División” football league case}

\begin{abstract}
This study examined the role that modern technology and social media play in sports. We focus on the Spanish top league Liga de Fútbol Profesional. The study found that the basic structure of sports communication stayed intact until recent years, but new technologies are causing deep changes. The last ten years can be viewed in sharp contrast to the preceding 75 years, as sports communication has been rapidly changing and will continue to do so due to technical innovations and the resulting interconnectedness of today's society.
\end{abstract}

Key words: Sports; communication; social media; football; Spain; technology.

\section{Referencia normalizada}

Sotelo González, J. (2012). Deporte y social media: el caso de la Primera División del fútbol español. Historia y Comunicación Social, Vol. 17, páginas 217-230.

Sumario: 1. Introducción; 2. Estado de la cuestión y objetivo de la investigación; 3. Metodología; 4. Los clubes de fútbol españoles en la Red; 5. Hablan los expertos; 6. Conclusiones. 7. Bibliografía.

\section{Introducción}

A lo largo de la historia, la sucesiva introducción de nuevas tecnologías ha operado como elemento generador de cambio social. La digitalización de la información y la convergencia de este fenómeno con el de su distribución en red han traído consigo una nueva Era del desarrollo humano de dimensiones históricas. Desde la aparición de Internet, y más concretamente con la llegada de la web 2.0, el mundo de la Comunicación ha experimentado grandes transformaciones, consoli- 
dando así la idea de que la historia de la comunicación está estrechamente vinculada a la historia de la tecnología. El modelo teórico lineal de comunicación masiva en el que un emisor (fuente) transmite un mensaje a través de un canal (medios) destinado a un receptor (público), durante muchos años paradigma dominante en los estudios sobre transmisión de información a través de los medios de comunicación social, admite hoy ciertas revisiones en favor de un modelo más reticular que lineal, con el que se ha pasado del tradicional flujo informativo básicamente unidireccional "de uno a muchos" a un flujo de corte multidireccional "de muchos a muchos". En definitiva, algunos viejos modelos explicativos de la comunicación de masas han sido ya superados por los acontecimientos y, tal como señala Carlos Scolari (2008: 123-127), los investigadores se reagrupan hoy en torno a cuatro paradigmas: el paradigma crítico, cuyos principales exponentes son Maldonado (1998), Adorno y Horkheimer (1998), Marcuse (2001) y Habermas (1998); el paradigma empírico-analítico, con las investigaciones de Castells (1996-1998) o Nielsen (1993, 2000); el paradigma interpretativo-cultural, encabezado por Marshall (2003), y el semiótico-discursivo, representado por Bettetini y Colombo (1993), por Del Villar (2004) o por el propio Scolari (2004).

Lo estrictamente tecnológico, con ser fundamental, no es el único elemento clave de este proceso que muchos califican ya como "cambio de paradigma" y en el que, como en todas las revoluciones, hay otro protagonista insoslayable: el individuo. La gran diferencia entre los nuevos medios y los medios tradicionales radica en el mayor potencial interactivo de estos últimos. Internet y el resto de nuevos medios sociales permiten el flujo multidireccional de mensajes y mayores posibilidades de selección, expresión y comunicación. Hay quien interpreta que Internet no es tanto una tecnología como una producción cultural, porque se trata de una construcción realmente social, en la que los usuarios son simultáneamente consumidores y productores de contenidos ("prosumidores"). Son las propias personas las que configuran este nuevo modelo reticular mediante sus aportaciones, mediante su participación activamente creativa y mediante sus actitudes colaborativas. Sheizaf Rafaeli (1988) apuntaba ya hace unos años que los medios de comunicación mejorarían si emulaban de algún modo la forma en que la gente se comunica en las conversaciones cara a cara.

Las máquinas por sí solas ni han sido ni son capaces de generar un discurso interactivo pleno, pero son herramientas necesarias para que los usuarios desarrollen sus capacidades de relación. El individuo ha cobrado así un gran protagonismo en la formación y el crecimiento de esta cultura de red interactiva que ha abierto como nunca antes la mentalidad humana al diálogo y al planteamiento de modo continuo en un escenario de participaciones múltiples. Según Alejandro Rost (2006: 233), los internautas "buscan dialogar, discutir, confrontar, apoyar y, de una u otra manera, entablar una relación con otros". En definitiva, por potente que sea, la tecnología tan solo es una herramienta habilitadora que cobra su verdadera fuerza en manos de la gente (conectada).

Tanto cuantitativa como cualitativamente hablando, la irrupción de los canales sociales online (social media) quizá sea el fenómeno más significativo acontecido en el ámbito de Internet en los últimos años, de modo que la interacción de personas en 
tiempo real, sin importar la localización de las mismas, se ha convertido en una de las actividades estrella del momento en el mundo online, algo de lo que algunos datos dan buena muestra: el $90 \%$ de los usuarios de Internet pertenecen ya a alguna red social; el social media ha superado ya al consumo de pornografía como primera actividad en la Red; uno de cada ocho matrimonios en Estados Unidos en 2010 se originó vía social media; el $80 \%$ de las empresas estadounidenses usan LinkedIn para reclutar empleados y, según el estudio "Seguridad y Privacidad en el uso de los Servicios Móviles por los menores españoles", publicado a finales de 2011 por el Instituto Nacional de Tecnologías de la Comunicación (INTECO) y la operadora Orange, la tasa de menores que accede a las redes sociales en Internet desde teléfonos móviles inteligentes se multiplicó casi por ocho entre 2010 y 2011, pasando de representar el 7\% del colectivo en 2010 al 54\% en 2011. Según ese mismo estudio, un $85 \%$ de los adolescentes españoles de entre 15 y 16 años que se conectan a Internet desde sus móviles lo hacen para acceder a redes sociales. Quizá prueba definitiva de la pujanza y de la exitosa implantación social de las últimas tecnologías y de fenómenos de uso asociados a ellas es la asombrosa celeridad con la que han triunfado, porque tal y como apunta Erik Qualman en su documental Social Media Revolution, para alcanzar los 50 millones de usuarios, la radio necesitó 38 años; la televisión precisó de 13 años; Internet, de 4 años; el iPod, de 3 años; y a Facebook le bastaron apenas nueve meses para alcanzar sus 100 primeros millones de usuarios.

Los últimos medios técnicos de comunicación han revolucionado la manera tradicional de ver el mundo. Ahora existe una balanza en la que, por un lado, tenemos la información estructurada y controlada que nos ofrecen los medios tradicionales $\mathrm{y}$, por otro, una acción colectiva ciudadana que genera contenidos propios y los coloca en plataformas de acceso teóricamente universal. Y lo mismo ocurre en los ámbitos institucionales: Internet ha facilitado que las empresas e instituciones creen sus propios "medios de comunicación", rompiendo así la tradicional diferencia de roles entre los medios que "informan a los demás" y los sujetos sociales de la información.

Situando ya el foco de estas reflexiones iniciales en el ámbito del deporte, es también indiscutible la influencia que sobre éste están teniendo las últimas tecnologías de la información y la comunicación, y viceversa. Para entender con visión holística la evolución del deporte a lo largo de la historia, es necesario atender paralelamente a los cambios experimentados por los medios de comunicación y a la propia evolución de las tecnologías de la información, porque la ósmosis entre deporte y comunicación va más allá del extremadamente importante papel que los medios de comunicación han cumplido - y cumplen- en la financiación de los deportes, fundamentalmente, aunque no exclusivamente, en su esfera profesional. Los medios han espectacularizado el deporte hasta el punto de moldear en los espectadores y en los propios deportistas una particular experiencia del mismo. Claude Sobry (2003: 10) dijo en su día que "ya no hay deporte sin televisión, ni televisión sin deporte". De Moragas (2000: 3), parafraseando a Dominique Wolton (1999), apuntaba ya en los albores del siglo XXI que en un contexto de profundos cambios en el sistema de producción y distribución de la comunicación, "el deporte se ha convertido en una práctica social representativa de los procesos de convergencia que 
se producen en la sociedad de la información entre los factores tecnológicos, culturales y sociales", y que esta convergencia "no debe ser considerada, pues, como un fenómeno aislado, sino como una consecuencia directa de las transformaciones que se están produciendo en el sistema de comunicaciones y en los nuevos procesos de mediación cultural en la sociedad de la información".

\section{Estado de la cuestión y objetivo de la investigación}

Durante los últimos veinte años, se ha hecho necesario un esfuerzo investigador orientado a cubrir el espacio generado por la influencia de las nuevas tecnologías y sus aplicaciones comunicativas, caracterizadas especialmente, como apuntábamos párrafos atrás, por sus capacidades interactivas. No obstante, y a pesar de la importancia que están cobrando los "medios sociales" online en la actualidad, existe poca teoría derivada de investigaciones empíricas para comprender el fenómeno de estos medios en el deporte, y menos aún, en el ámbito más concreto del fútbol español. El ciberespacio, en general, sigue presentándose como un nuevo campo de investigación donde también se precisan metodologías científicas, tanto para la comprensión de las claves de la infosociabilidad como para la elaboración de nuevas teorías o para ampliaciones conceptuales de teorías preexistentes.

La simbiótica relación del deporte con la comunicación, lato sensu, ha sido abordada desde numerosas perspectivas y existe ya una relativamente abundante literatura científica al respecto, pero el ángulo de ataque que aquí proponemos carece de antecedentes relevantes, por lo que con la presente aportación también tratamos de cubrir cierto vacío investigador en un ámbito apenas tratado con el rigor, la profundidad y la sistematicidad que reclama y, a nuestro parecer, merece.

\section{Metodología}

Para la producción del presente trabajo, hemos recurrido a tres técnicas de investigación de uso común y aceptado en el ámbito de la comunidad científica: la revisión bibliográfica, el análisis de contenido (de páginas web, en nuestro caso) y el juicio de expertos.

La revisión bibliográfica y documental constituye uno de los principales pilares en los que se sustenta toda investigación. Por una parte, nos ha facilitado la elaboración del marco teórico y, por otra, nos ha permitido delimitar con mayor precisión nuestro objeto de estudio y constatar el estado de la cuestión. Esta técnica nos ha resultado especialmente útil a la hora de enmarcar nuestro objeto de interés en la introducción. Para ello, hemos buscado información relevante de actualidad en español y en inglés en libros, artículos e Internet, otorgando siempre atención preferente a las fuentes de documentación primarias (textos completos y originales) frente a las secundarias (aquellas que seleccionan, referencian o resumen la información primaria). Existe una muy escasa (por no decir nula) literatura científica al respecto. La mayor parte de documentos pertinentes encontrados (casi todos en Internet) son 
informes de consultorías y escritos técnicos o profesionales de baja visibilidad pública.

El análisis de contenido de las páginas web de los 20 clubes de la Primera División española de fútbol en la temporada 2011-2012 ha sido realizado mediante la creación de categorías de análisis para la sistematización de los contenidos manifiestos en esas webs. El procedimiento ha sido básicamente descriptivo y hemos prestado especial atención a los elementos de social media presentes en nuestra población de estudio. La valoración de las sedes web ha sido realizada durante los diez últimos días del mes de enero de 2012. El análisis de la presencia de los antedichos clubes en redes sociales se llevó a cabo también durante los últimos diez días de enero de 2012, aunque el seguimiento del fenómeno de las redes sociales online, en general, y de éstas en relación con el deporte, en particular, viene siendo una de nuestras líneas preferentes de investigación y trabajo desde 2010.

Por último, a través de las opiniones de varios expertos en la materia de nuestro interés obtenidas mediante entrevistas personales y vertidas también en los antedichos informes de consultorías y escritos técnicos y profesionales, hemos recabado un conjunto de opiniones brindadas al respecto de nuestro objeto de estudio por reputados especialistas en dicho objeto. La ventaja de esta herramienta metodológica radica fundamentalmente en el profundo conocimiento que suelen aportar los expertos sobre los temas objeto de evaluación. Además, se trata de una técnica de investigación que permite la flexibilidad y la adaptación ante las diferentes situaciones que puedan surgir durante la evaluación. Este recurso comporta costes reducidos, un notable ahorro de tiempo respecto a otras técnicas y confiere una gran credibilidad a las conclusiones que con él se obtienen.

\section{Los clubes de fútbol españoles en la Red}

Los 20 clubes de la Primera División del fútbol español en la temporada 20112012 analizados contaban en el momento de nuestro estudio con páginas web oficiales. El contenido preferente de todas ellas es, obviamente, la información relacionada con cada equipo: estado de los jugadores, fichajes, resultados anteriores, próximos partidos, testimonios, alineaciones y partes médicos. El $75 \%$ de los equipos ( 15 de los 20 analizados) utilizan formatos de vídeo para transmitir información, el resto utiliza formatos de texto. Otro de los elementos comunes al 75\% de los sitios web estudiados es la presencia de tienda online para la comercialización de productos del club, porcentaje que desciende al 60\% (12 clubes de los 20 ) cuando se trata de venta de entradas online (bien sea a través de plataforma propia o mediante enlace a otras plataformas externas, como, por ejemplo, www.entradas.com). Las campañas promocionales para la captación de nuevos socios (adaptando las utilizadas en otros medios o mediante banners creados específicamente para sus webs) es otro de los recursos generalizados en el $70 \%$ de las 20 webs analizadas. En cuanto a acciones de marketing móvil, 8 de los 20 clubes ofrecen en sus webs aplicaciones para teléfonos móviles. Prácticamente la totalidad de las webs de los clubes analizadas, excepto 3 , ofrecen algún tipo de material multimedia de potencial interés para 
los aficionados, como fondos de escritorio, salvapantallas, sonidos para el móvil o newsletters. Dejando aparte las encuestas y las porras que proponen en sus webs varios clubes, solamente 8 de ellos incluyen en sus sitios online oficiales un club de fans para promover cierta participación real de esos fans (y no únicamente una participación simbólica, decorativa o circunstancial) de todos sus simpatizantes (no solo socios). La totalidad de los clubes enlazan desde la página principal de su web (home) a otros sitios sociales, principalmente, a Facebook y a Twitter.

Tal como muestra el cuadro ${ }^{\circ} 1$, el $100 \%$ de los clubes de la Primera División de la LFP (Liga de Fútbol Profesional) en la temporada 2011-2012 tenían presencia en alguna red social. Diecisiete de ellos cuentan con presencia en Twitter, red a la que enlazan desde las páginas principales de sus webs, lo que hace presuponer que se trata de cuentas oficialmente reconocidas, no así en los casos de Racing de Santander, Real Zaragoza y Getafe CF, donde se hace complicado diferenciar si sus perfiles de Twitter encontrados al rastrear la web son oficiales o no (apreciamos que no lo son, por lo que no hemos marcado la correspondiente casilla en el cuadro $\mathrm{n}^{\mathrm{o}} 1$ ). Cabe destacar que a fecha 23 de enero de 2012, el 20\% de los equipos analizados no tenía página oficial en Facebook, plataforma social líder en el mundo, con más de 600 millones de usuarios. Por otra parte, únicamente 7 de los 20 clubes (35\%) enfoca parte de su comunicación en redes online hacia los más jóvenes a través de la plataforma Tuenti, red con un perfil de usuario mayoritariamente juvenil y adolescente. Únicamente tres clubes disponían a finales de enero de 2012 de cuenta oficial en Google +, la red social que el gigante Google lanzó a mediados de 2011. Las promociones y la información corporativa aglutinan la mayor parte de la actividad de los equipos en las redes online.

Cuadro $n^{\circ}$. Presencia de los equipos de fútbol de Primera División en las redes sociales.

\begin{tabular}{|l|c|c|c|c|c|c|c|}
\hline & Facebook & Twitter & Tuenti & Google + & YouTube & RSS & Otros canales \\
\hline FC Barcelona & $\mathrm{X}$ & $\mathrm{X}$ & & $\mathrm{X}$ & $\mathrm{X}$ & $\mathrm{X}$ & $\begin{array}{c}\text { Barça Fans } \\
\text { Weibo }\end{array}$ \\
\hline Real Madrid & $\mathrm{X}$ & $\mathrm{X}$ & $\mathrm{X}$ & & $\mathrm{X}$ & $\mathrm{X}$ & $\begin{array}{c}\text { Renren } \\
\text { Weibo }\end{array}$ \\
\hline Valencia CF & $\mathrm{X}$ & $\mathrm{X}$ & $\mathrm{X}$ & & & $\mathrm{X}$ & \\
\hline Villarreal CF & $\mathrm{X}$ & $\mathrm{X}$ & & & & $\mathrm{X}$ & Myvillarreal \\
\hline Sevilla CF & $\mathrm{X}$ & $\mathrm{X}$ & $\mathrm{X}$ & $\mathrm{X}$ & $\mathrm{X}$ & $\mathrm{X}$ & \\
\hline Athletic Club & $\mathrm{X}$ & $\mathrm{X}$ & & $\mathrm{X}$ & & & \\
\hline Atlético Madrid & $\mathrm{X}$ & $\mathrm{X}$ & $\mathrm{X}$ & & $\mathrm{X}$ & $\mathrm{X}$ & \\
\hline RCD Espanyol & $\mathrm{X}$ & $\mathrm{X}$ & $\mathrm{X}$ & & $\mathrm{X}$ & $\mathrm{X}$ & \\
\hline CA Osasuna & $\mathrm{X}$ & $\mathrm{X}$ & & & & & \\
\hline R. Sporting G. & $\mathrm{X}$ & $\mathrm{X}$ & & & & & \\
\hline Málaga CF & $\mathrm{X}$ & $\mathrm{X}$ & $\mathrm{X}$ & & $\mathrm{X}$ & $\mathrm{X}$ & \\
\hline R. Racing S. & & & & & $\mathrm{X}$ & $\mathrm{X}$ & \\
\hline Real Zaragoza & & & & & & & \\
\hline Levante UD & $\mathrm{X}$ & $\mathrm{X}$ & & & $\mathrm{X}$ & $\mathrm{X}$ & \\
\hline Real Sociedad & $\mathrm{X}$ & $\mathrm{X}$ & & & & $\mathrm{X}$ & \\
\hline Getafe CF & & & & & & & \\
\hline RCD Mallorca & $\mathrm{X}$ & $\mathrm{X}$ & & & & & \\
\hline Real Betis & $\mathrm{X}$ & $\mathrm{X}$ & $\mathrm{X}$ & & $\mathrm{X}$ & & \\
\hline Rayo Vallecano & & & & & $\mathrm{X}$ & $\mathrm{X}$ & \\
\hline Granada CF & $\mathrm{X}$ & $\mathrm{X}$ & & & & & \\
\hline
\end{tabular}

Fuente: Elaboración propia 
Por lo que se refiere a la presencia de clubes en la red social líder en el mercado (Facebook), el conjunto de los equipos analizados sumaban cerca de 50 millones de fans en ella a finales de enero de 2012. En este apartado, podrían identificarse tres grupos de equipos en función del número de seguidores. Por una parte, el FC Barcelona, con más de 25 millones de fans de su página, y el Real Madrid, con cerca de 23 millones y medio de seguidores. En un segundo nivel, se situarían todos aquellos equipos con perfil oficial en Facebook (dejando aparte las consideraciones sobre las notables oscilaciones en el número de seguidores de cada uno de ellos). Por último, un tercer grupo de tres equipos sin presencia oficial en Facebook. Doce de los 20 clubes $(60 \%)$ con presencia en Facebook utilizan esta red como herramienta de marketing online diariamente. En general, casi todos los equipos utilizan Facebook como canal para emitir noticias de manera unidireccional, de modo que únicamente Villarreal CF y Real Betis responden a los comentarios de sus seguidores de manera habitual. A fecha de realización del trabajo de campo, solo el Sevilla CF y el Atlético de Madrid realizaban promociones a través de Facebook.

Según la revista electrónica mensual alemana Social Media and Sport (número 9, octubre de 2011), la Liga de Fútbol española es de las más activas de Europa en términos de número absoluto de seguidores online de sus clubes, solo por detrás y a muy poca distancia (en octubre de 2011) de la Premier League británica y muy por delante del resto de Ligas de fútbol europeas. Además, los porcentajes de crecimiento mensuales de la Liga española están entre los 5 mayores de las 18 Ligas europeas analizadas por el Observatorio que edita esta revista. A nivel de clubes, y según esta misma fuente, también son dos equipos españoles -Real Madrid y FC Barcelona- los que más seguidores tenían en Facebook a octubre de 2011 (alrededor de 20 millones cada uno), seguidos muy de cerca por el Manchester United (19 millones y medio de seguidores a octubre de 2011), pero muy por encima del resto de grandes clubes europeos. Sin duda, estas dos gigantes marcas globales -Real Madrid y FC Barcelona- por sí solas constituyen la locomotora estadística de todo el conjunto de clubes españoles. Únicamente el Málaga CF, impulsado quizá por sus nuevos dueños y por la incorporación a su plantilla de jugadores de mucho renombre, ha presentado en los primeros meses de la temporada 2011-2012 crecimientos porcentuales superiores a los de Real Madrid y FC Barcelona, que en términos absolutos, resultan claramente inalcanzables. Según Social Media and Sport, el perfil oficial del Málaga CF en Facebook pasó de contar con 177.725 seguidores a 1 de septiembre de 2011 a contar con 257.229 seguidores justo un mes después (un $44,73 \%$ de crecimiento en 30 días).

Respecto al uso de Twitter, todos los clubes que lo utilizan lo actualizan prácticamente a diario para emitir las mismas noticias que ofrecen en sus webs y también para narrar los partidos. El club que cuenta con más seguidores (followers) en este canal es el Real Madrid, con 3249.446 seguidores; seguido de lejos por el FC Barcelona, con 11672.718 seguidores; el Valencia CF, con 73.442 seguidores y el Sevilla CF, con 33.779 seguidores. De manera similar a como sucede con Facebook, solo dos clubes, Real Madrid y Villarreal CF, conversan habitualmente con sus seguidores y responden a los comentarios de estos en Twitter, mientras que Valencia CF y 
Sevilla CF lo hacen ocasionalmente. Precisamente estos dos clubes son los únicos que ofrecen promociones a través de este canal. Curiosamente, todos los clubes cuentan con miles de seguidores en Twitter, pero los equipos apenas siguen a otros usuarios, contrariamente a la dinámica propia y característica de esta red social, y cuando lo hacen, se trata de sus propios patrocinadores o de empresas, instituciones o personas muy vinculadas al club. El FC Barcelona únicamente sigue a 34 usuarios, y el Real Madrid, a 20.

Hemos contabilizado que 10 clubes tienen canal propio en YouTube enlazado desde sus webs oficiales: FC Barcelona, Real Madrid, Sevilla FC, Atlético de Madrid, RCD Espanyol, Málaga CF, Racing de Santander, Levante UD, Real Betis y Rayo Vallecano. Más son aún los equipos (12 de 20) que ofrecen en sus webs la posibilidad de sindicación mediante RSS. Únicamente 2 clubes, RCD Mallorca y Athletic Club de Bilbao, cuentan con blogs.

En el apartado "otros canales", destaca (pero no sorprende) la presencia de los dos principales clubes de fútbol españoles en el mercado asiático de redes sociales, una presencia lógica si tenemos en cuenta los fuertes intereses de ambos clubes en el mercado asiático en general. Tanto el Real Madrid como el FC Barcelona están presentes en Weibo, la versión china de Twitter. El Real Madrid dispone también de perfil oficial en Renren, un gigante chino del social media que cotiza en la Bolsa de Nueva York y que a principios de 2011 contaba con 160 millones de usuarios registrados y 31 millones de usuarios activos por mes. Otra característica muy interesante que demuestra la globalización del fútbol y, en particular, de estos dos grandes clubes, es el hecho de que ambos han creado cuentas de Twitter en diferentes idiomas. El FC Barcelona, en castellano, catalán e inglés; y el Real Madrid, además de en castellano e inglés, en japonés y en árabe. Por último, en lo relativo a "otros canales", destacar que FC Barcelona y Villarreal CF han creado sendas comunidades virtuales propias: Barça Fans y Myvillarreal, respectivamente.

\section{Hablan los expertos}

A pesar de que los medios sociales online son ya un fenómeno masivo y creciente en casi todos los ámbitos de la actividad humana, gran parte de los expertos españoles en marketing deportivo apuntan que el fútbol profesional español es aún un gran underperformer en todo lo relacionado con los nuevos medios y, especialmente, en las redes sociales, porque como apunta Javier Fernández de la Cruz, responsable del departamento de marketing online en Sowre Consulting España, incluso aquellos que utilizan el marketing online y las redes sociales de manera habitual, "lo hacen de forma casi unidireccional, cuando la verdadera fortaleza de las redes sociales es la conversación y la bidireccionalidad, poniendo al usuario al mismo nivel que la organización". Es la apreciación que también destaca Stephan van Uijtregt, consultor en innovación deportiva en Nuevola:

A pesar de la amplia variedad de herramientas y aplicaciones que están disponibles para este diálogo, la comunicación en este momento es casi exclusivamente sentido 
único. Por supuesto hay excepciones positivas, por ejemplo, Jorge Lorenzo, que mantiene una línea abierta con su base de fans a través de Twitter. Muchos deportistas españoles han conocido los nuevos medios [...] y espero verles usarlos de una manera social.

Para Eric Serra, de Sports Marketing \& Communications, aunque los medios sociales se están abriendo camino poco a poco en Europa, se da en muchos casos un uso 1.0 de estos canales, con una actividad en los mismos "muy poco natural", como la no participación de algunas estrellas del deporte o de los propios clubes de fútbol en la conversación online o que estos se limiten a lanzar información o titulares al estilo de la prensa escrita. En este sentido, Eric Serra coloca en la vanguardia al deporte americano:

Cuando ves el deporte americano en redes sociales como Twitter o Facebook, te das cuenta que en Europa vamos dos pasos por detrás. Organizaciones profesionales, equipos, jugadores, periodistas... han conseguido entre todos hacer participar a todo el mundo dentro de la rueda que hace girar el deporte cada día, un showtime permanente de declaraciones, vídeos, en definitiva, conversaciones que en Europa siguen siendo poco comunes.

El juicio mayoritariamente extendido entre los expertos en materia de social media en el fútbol profesional español es que se echa en falta una mayor participación social online en la vida de los clubes y que aún faltan etapas para conseguir que sean clubes realmente 2.0. Miquel Pellicer, jefe de contenidos y community manager de Mundo Deportivo, y Jordi Andreu, de Esendex, coinciden al señalar que hoy en día parece no existir una estrategia clara para redes sociales a nivel de marketing deportivo, de manera que para Jordi Andreu, "los clubs, deportistas y organizaciones deportivas no están sacando partido al poder de comunicación bidireccional, de fidelización, captación y relación con los fans", llaga sobre la que también pone el dedo Miquel Pellicer:

Maximizar los procesos de participación de socios y simpatizantes y convertir las entidades deportivas en organizaciones más participativas y transparentes debería ser el objetivo del Social Media aplicado en el mundo del deporte. Los tiempos del espectador pasivo han finalizado y pisa con fuerza el individuo que opina $\mathrm{y}$, sobre todo, que quiere formar parte de unos colores. De la misma forma, los tiempos de la gestión deportiva sin escucha activa deberían pasar a la historia.

Para Eneko Ruiz Alfaro, community manager en BKOOL, el problema es una vez más cómo monetizar las bases de fans y cómo sacar rendimiento económico directo de las redes sociales. Y quizá sea en este aspecto donde radica parte de la explicación a esos márgenes para la mejora que aún quedan en la implantación y desarrollo del social media en el fútbol profesional español, un "negocio" que todavía encuentra sus principales fuentes de ingreso en partidas más tradicionales, especialmente, en la venta de derechos televisivos. 
El 22 de mayo de 2011, el suplemento dominical Negocios, de El País, publicaba un artículo en el que, bajo el título "Más que un club, una marca global" y a propósito del crecimiento económico experimentado por el FC Barcelona durante los últimos años, se ofrecían algunos interesantes datos sobre la procedencia de los ingresos de los principales clubes. En el caso del FC Barcelona, al igual que sucede con ligeras variaciones con muchos de otros grandes clubes europeos, Real Madrid incluido, los derechos de radiodifusión le aportaron en 2010 al club catalán 178,1 millones de euros, sobre un montante total de ingresos de 398,1 millones de euros en ese mismo año, lo que supone que un $44 \%$ del total de ingresos proviene de la partida "radiodifusión", tal como muestra la Figura 11.

Figura 1.

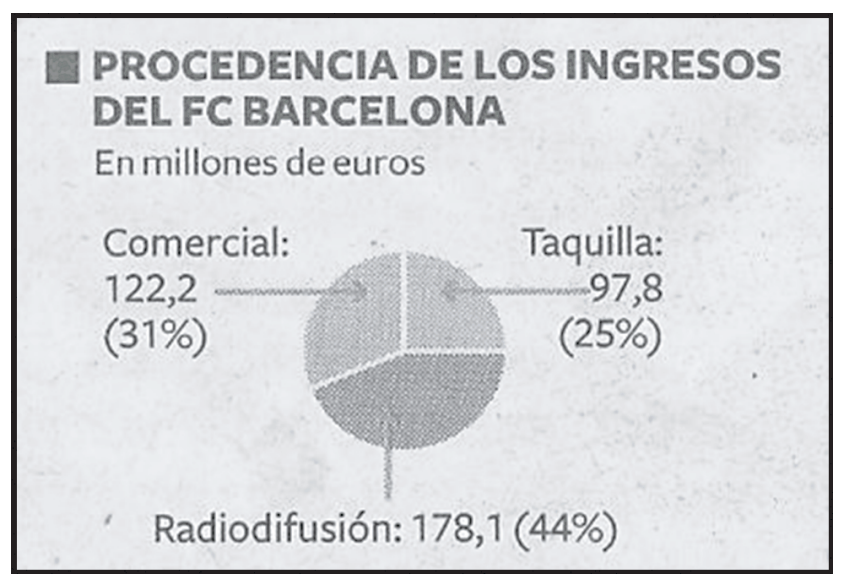

Fuente: El País (Negocios, 22/05/2011, p. 16)

No obstante, Eneko Ruiz Alfaro confía en que en el inmediato futuro haya más clubes y entidades que utilicen el social media en clave comercial y que la geolocalización permita mejorar la activación del patrocinio, la venta de entradas y otras fórmulas de comercialización de productos y servicios. También apunta que respecto a los derechos de televisión,

el baloncesto ha dado un paso adelante en este aspecto a nivel mundial con la retransmisión en live streaming en abierto de partidos y espero que se sumen cada vez más deportes. Los patrocinadores de los clubes podrán disfrutar de un impacto nacional e internacional que antes solo les permitía la televisión. Para las ligas, los patrocinadores de los partidos suplirán los ingresos de los clásicos pay-per-view que siguen dándose en algunas ligas.

Dídac Lee, consejero delegado de Inspirit y actualmente en la directiva del FC Barcelona presidida por Sandro Rosell, también opina que la retransmisión de eventos en directo vía streaming a través del vídeo será cada vez más habitual, aunque condicionada por los contratos vigentes de distribución de contenidos. Más 
que en lo técnico, el problema radicará, según este emprendedor, en la disponibilidad de plataformas digitales por parte de los propietarios de los derechos y de que la nueva línea de distribución esté alineada con su estrategia de negocio.

Para Joan Moreno, community manager de Betfair en España, todos los clubes acabarán explotando las redes sociales a instancia en muchos casos de sus anunciantes, entre ellos, las pujantes casas de apuestas online, como la propia Betfair. El año 2011 ha sido muy prolífico en noticias sobre jugadores o equipos que se decidían a gestionar en las redes online su identidad digital en primera persona o a través de alguna marca. Las grandes estrellas y buena parte de las entidades deportivas ya tienen presencia en Facebook o Twitter, desde donde incluso ofrecen ruedas de prensa. Los clubes ya no solo están de forma testimonial en las redes sociales online, sino que forman parte de ellas y se han hecho necesarias las alianzas entre el club como marca y el poderoso personal branding de los deportistas del club. Cada vez son más numerosas las estrategias y acciones específicas de los clubes en redes sociales: información, promociones, servicio al cliente, comercialización, etc. Para Josep M. Monti, director ejecutivo y fundador de Esforc, hablar de redes sociales ya no es ninguna novedad, "si no estás en ellas no existes y punto". Los clubes más destacados ya tienen sus propias aplicaciones (apps) para permitir acceder desde el móvil a los contenidos multimedia que ofrecen.

A propósito del personal branding, resulta interesante y hasta cierto punto paradójico ver que si a nivel de club el FC Barcelona va cuantitativamente un paso por delante del Real Madrid, los jugadores estrella de este último tienen más seguidores que los del FC Barcelona. Cristiano Ronaldo, por ejemplo, tenía a principios de febrero de 2012 alrededor de 39 millones de fans en Facebook, casi 9 millones más que la estrella del FC Barcelona, Leo Messi, en esas mismas fechas. Y las diferencias se agudizan más si tomamos como referencia el conjunto de jugadores de uno y otro equipo. Quizás esto también venga a confirmar la diferencia de filosofías de club, que en el caso del FC Barcelona sitúa a la entidad como lo más importante, mientras que el Real Madrid, fiel a su esencia, basa gran parte de su popularidad en las estrellas que lo integran.

Respecto al inmediato futuro del social media en general, muchos expertos coinciden en apuntar al desarrollo de la geolocalización, de la realidad aumentada y del real time como tres de los puntos clave. La geolocalización cobrará mayor presencia en el marketing deportivo y seguirán apareciendo nuevos servicios que geoposicionarán en tiempo real contenidos de todo tipo vinculados a los eventos deportivos. El real time, en el que basan gran parte de su fuerza las redes sociales online, permitirá explotar el poder del directo de forma alternativa (o complementaria) a la cobertura tradicional de eventos deportivos. Plataformas como Twitter o Facebook podrían crear adaptaciones específicas en su entorno (social TV), como ya hicieron con el seguimiento del Mundial de Fútbol de Sudáfrica en 2010.

Casi todos los medios periodísticos ya cuentan con perfil en Twitter y los aficionados se están enterando por esta red de los goles y las noticias deportivas antes que en ningún otro medio. Pero el éxito de esta forma de comunicación viene dado principalmente en que cualquier persona se convierte en emisora de noticias. ¿Qué nos 
deparará ello? ¿Poder seguir un partido desde las imágenes captadas por los aficionados? ¡Miles de cámaras emitiendo en directo desde los estadios! O también, por qué no, seguir el derbi entre los equipos locales del barrio (Josep M. Monti).

El mobil gaming, o más en general, todo lo relacionado con el marketing orientado a dispositivos móviles, es otro de los grandes nichos de mercado para el social media. Cristiano Ronaldo (no como persona, sino como marca) se sirvió de su gran número de seguidores en Twitter, Facebook y TenCent para promocionar su popular juego para iPhone Heads Up with Cristiano y colocarlo entre las descargas más solicitadas en la Apple Store. El aumento del número de contenidos publicados en los medios con origen en las redes sociales, la aparición de comunidades más específicas o verticales donde los usuarios compartan aficiones o intereses comunes y el refinamiento de los sistemas y procedimientos de medición de los fenómenos online serán también, según los expertos, algunas de las grandes líneas del futuro inmediato en el social media deportivo.

\section{Conclusiones}

El uso y la explotación de las posibilidades que ofrece el mundo digital 2.0 no están tan implementados en el deporte como creemos. La principal conclusión que se puede sacar del estudio de las webs de los clubes de fútbol españoles de Primera División en la temporada 2011-2012 es que se utilizan como canal de comunicación unidireccional, ofrecen noticias del club y comercialización de productos y servicios, pero permiten aún poca interacción por parte de los aficionados. Y lo mismo sucede con su presencia en las redes sociales online, utilizadas por las marcas, instituciones y deportistas de manera básicamente unidireccional, como canales para la propagación de sus comunicados offline. Muy pocos utilizan las redes allí donde son más potentes: para conversar con los seguidores. En líneas generales, los primeros clasificados (deportivamente hablando) al término de la pasada Liga 2010-2011, están también en cabeza en lo que respecta a uso de redes sociales y otras herramientas de comunicación y marketing online.

En conjunto, aunque se aprecia el interés de los clubes por incorporarse a las nuevas plataformas de comunicación, la gran mayoría todavía no tiene bien definidas sus estrategias online, lo que les hace perder oportunidades a la hora de crear experiencias significativas para una comunidad de aficionados y seguidores muy activos en las redes sociales cuando se trata de demostrar su pasión por sus equipos. Muy pocos clubes dinamizan los canales sociales online con criterio y, en conjunto, se llevan a cabo pocas acciones para activar una participación real, efectiva y estable de sus seguidores o para premiar ese afán participativo de los fans.

En las redes sociales online se confirma también el fanatismo que existe en España por el fútbol $\mathrm{y}$, de nuevo, se hace patente el tópico de que este deporte "mueve masas": el conjunto de los equipos de Primera División de la Liga 20112012 sumaban cerca de 50 millones de seguidores solo en Facebook a principios de 2012. Si comparamos estos datos con el número de socios, encontramos, por 
ejemplo, que por cada socio del Real Madrid (91.650), tenemos 257 fans en Facebook (23.585.937). Y si en las redes sociales online se confirma el fanatismo que existe en España por el fútbol, también viceversa: el mundo del fútbol ha confirmado, a su vez, la exitosa y espectacular implantación social del fenómeno de las redes sociales online.

La enconada rivalidad entre los dos grandes clubes del fútbol español, Real Madrid y FC Barcelona, también tiene su reflejo en el mundo online. Un ejemplo de ello es que los dos clubes han incluido de forma "sospechosamente" similar en sus sitios web marcadores de número de seguidores en las dos principales redes sociales online, Facebook y Twitter. Aunque gran parte de los expertos reconocen que el Real Madrid tiene una estrategia de contenidos más compacta, el FC Barcelona va por delante en términos de actividad generada por sus aficionados.

Los datos estadísticos en análisis de social media son espectacularmente volátiles. Equipos como el Real Madrid o el FC Barcelona pueden ganar seguidores en Facebook, como de hecho ha ocurrido durante el segundo semestre de 2011, a ritmo de un millón más de seguidores cada mes. En este sentido, la existencia de un observatorio de análisis periódico sería muy conveniente y de gran utilidad a la hora de evaluar trayectorias y de proyectar tendencias en el caso español.

Aunque se observan excepciones, en general, existe una correlación entre el número de seguidores online de los clubes y la posición deportiva ocupada por estos en la clasificación en la Liga, de lo que se deduce que los clubes rentabilizan en medios sociales su importancia clasificatoria como equipo.

Analizando los sitios web de los clubes de fútbol españoles de Primera División en la temporada 2011-2012, y cruzando esa labor con la de la búsqueda de documentación relevante al respecto, hemos encontrado cierta "asignatura pendiente". En este sentido, sería interesante incrementar el caudal de investigación rigurosa en materia de presencia online del fútbol español.

\section{Bibliografía}

MORAGAS SPÀ, M. de (2000). "Olimpismo, comunicación y cultura" [artículo en línea]. Barcelona: Centre d'Estudis Olímpics UAB. [Consultado el: 14/12/2011] [Disponible en http://olympicstudies.uab.es/pdf/wp095_spa.pdf]

RAFAELI, S. (1988). "Interactivity: From new media to Communications". En HAWKINS, R. P.; WIEMANN, J. M.; PINGREE, S. (eds.) (2001). Advancing communication science: Mergin Mass and interpersonal process. Newbury Park: Sage Publications.

ROST, A. (2006). La interactividad en el periódico digital. Tesis doctoral. Barcelona: Universidad Autónoma de Barcelona. [Disponible en http://www.tesisenxarxa.net/ TDX-1123106-104448/]

SCOLARI, C. (2008). Hipermediaciones. Elementos para una Teoría de la Comunicación Digital Interactiva. Barcelona: Editorial Gedisa.

SOBRY, C. (2003). Socioéconomie du sport. Structures sportives et liberalismeéconomique. Brusells: Éditions De Boeck Université. 
Social Media and Sport, $\mathrm{n}^{\circ} 9$ (October 2011), Munich: University of Applied Science Erding \& RESULT Sports.

WOLTON, D. (2000). Internet, ¿y después? Barcelona: Editorial Gedisa.

\section{Notas}

1 Para profundizar en el estudio de los principales modelos europeos de explotación de los derechos de retransmisión televisiva del fútbol, consultar GARCÍA SANTAMARÍA, J. V. (2011). "Derechos de fútbol y retransmisiones deportivas en Europa". En Revista Telos (Cuadernos de Comunicación e Innovación), $\mathrm{n}^{\mathrm{0}}$ 86, Madrid: Fundación Telefónica, pp. 1-11.

\section{El autor}

Joaquín Sotelo González es profesor de Periodismo en la Universidad Complutense de Madrid. Ha desarrollado estancias de docencia e investigación en Oxford University y el Instituto de Estudios Europeos de Florencia. Es miembro activo del grupo de investigación Observatorio de Medios Informativos, de la UCM. Entre sus diversos escritos, cuenta con varios dedicados a dos de sus pasiones: la comunicación y el deporte. 